\title{
IMPROVEMENTS ON THE ESTIMATE OF SEISMIC CHARGES
}

\author{
Simón Gershanik
}

1. Assuming that buildings behave under seismic accelerations as vertical clamped bars, that the accelerations are only horizontal and of a translatory character, we can put for the seismic charges q:

$$
q=\rho S\left(\frac{\partial^{2} u}{\partial t^{2}}+\frac{\partial^{2} X_{\mathrm{B}}}{\partial t^{2}}\right)+2 \varepsilon^{\prime} \frac{\partial u}{\partial t}
$$

the meaning of simbols being as follows:

$$
\begin{aligned}
& f \text { the mean density of the building } \\
& S \text { its mean transverse section } \\
& " \text { its deformation (fig. } 1 \text { ) } \\
& X_{13} \text { horizontal ground displacement at the } \\
& \text { place } B \text { where the building is champed } \\
& t \text { time, and } \\
& \varepsilon^{\prime} \text { a damping coefficient of the huildings. }
\end{aligned}
$$

Therefore $q$, due to a ground acceleration $\frac{\partial^{2} X_{B}}{\partial t^{2}}$ can be determined if we know the expression of $u$.

To get $u$ three different assumptions may he considered: $a$ ) the deformations are principally due to shear; $b$ ) they are principally due to flexure; and $c$ ) they are due to both causes. This paper deals only with assumptions $a$ ) and $b$ ).

a) In this case, as is well known, $u$ is given by the differential equation :

$$
\frac{\partial^{2} u}{\partial z^{2}}=k \frac{\partial^{2} u}{\partial t^{2}}+2 \varepsilon_{1} \frac{\partial u}{\partial t}+k \frac{\partial^{2} X_{\mathrm{B}}}{\partial t}
$$


(where $k=\frac{\rho}{\mu} ; \varepsilon_{1}=\frac{\varepsilon^{\prime}}{u S} ; \mu$ rigidity modulus) with the following houn. dary conditions

$$
\begin{array}{lll}
u=O & \text { when } & z=O \\
\frac{\partial u}{\partial z}=O & \text { when } & z=l
\end{array}
$$

b) Deformation $u$ is in this case given lyy:

$$
\frac{\partial^{4} u}{\partial z^{4}}=c \frac{\partial^{n} u}{\partial t^{2}}+2 \varepsilon_{2} \frac{\partial u}{\partial t}-c \frac{\partial^{2} X_{1}}{\partial t^{2}}
$$

where $c=\frac{\rho S}{E J}: \quad \varepsilon_{2}=\frac{\varepsilon^{\prime}}{E J}, E=$ elasticity modulus and $J$ moment of inertia of $S$ refered to the neutral axis of the luuilding. The boun dary conditions now are:

$$
\begin{aligned}
u=0 ; \frac{\partial u}{\partial z}=0 & \text { when } z=0 \\
\frac{\partial^{2} u}{\partial z^{2}}=0 ; \frac{\partial^{3} u}{\partial z^{3}}=0 & \text { when } z=l
\end{aligned}
$$

Assuming that huilding are at rest until seismic accelerations begins, we have in addition, that equations [2] and [4] must satisfy the initial conditions

$$
u=0, \quad \frac{\partial u}{\partial t}=0 \quad w h n t=0
$$

2. - Equations [2] and [4] have the form

$$
\frac{\partial^{n} u}{\partial z^{n}}=-r(z)
$$

If we admit that the second member is a continuous function of $z$ in the interval $O<z<l$ (l height of the luuilling) then, according to the theory of Green's functions, we may put the solution of [6] and therefore the solutions of [2] and [4] too, in the form (1) $\left({ }^{2}\right)$ :

$$
u=\int_{0}^{1} K(z, \check{\zeta}) r(\zeta) d \xi
$$


$K(z, \zeta)$ being Green's function of the equation

$$
\frac{\partial^{n} u}{\partial z^{n}}=0
$$

As $n$ is an even number and the boundary equations are homogeneous, Green's $K$ function should be symetrical with respect to $z$ and $\zeta$. On the other band $K(z, \zeta)$ may be considered as a kernel of the integral equation of second kind

$$
v(z)=\lambda \int_{0}^{1} K(z, \zeta) v(\zeta) d \zeta
$$

From this, and from [7] it follows that the solution of [6] is

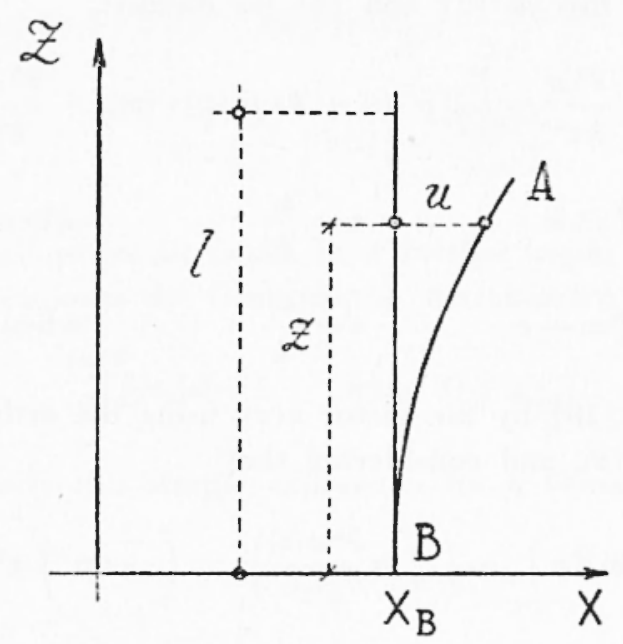

Fig. 1

a function represented according to the source $r(5, b y$ means of the symetrical kernel $K$. By Hilberts expansions theorem $\left({ }^{3}\right)$ it can, consequently be expanded in an uniformly convergent series of eigenfunctions $v(z)$ of this kernel.

'There fore we may write

$$
u=\sum_{\mathrm{j}=1}^{\mathrm{J}=\bar{p}_{\mathrm{j}} \mathrm{N}} v_{\mathrm{j}}(z)
$$

$v_{2}(z)$ being such eigenfunctions, and $p_{\mathrm{J}}$ coefficients independent of $z$. 
As $u$ is a function of $t$, we can differentiate the expression [7] with respect to this variahle. We then gret expressions similar to [\%]; from this it follows that the two derivates my also be expanded in uniformly convergent series of $v(z)$.

By interration of the series representing $\frac{\partial^{2} u}{\partial t^{2}}$ with respect to $t$. we obtain another representing $\frac{\partial u}{\partial t}$, and by integration of this wc
obtain another representing $u$. This, as well as the fact that $u$ is given by [8], allows us to write:

$$
\frac{\partial u}{\partial t}=\sum_{\mathrm{j}=t}^{j} p_{\mathrm{j}}^{\prime}(t) v_{\mathrm{j}}(z): \frac{\partial^{*} u}{\partial t^{\circ}}=\sum_{\mathrm{j}=1}^{j=\sum_{\mathrm{j}}^{2}} p^{\prime \prime} v_{\mathrm{j}}(t)
$$

Substituting this in [2] and [4] we obtain:

$$
\left.\frac{1}{M} \frac{\partial^{n} u}{\partial z^{n}}=\sum_{j=1}^{\infty}\left[p^{\prime \prime}(t)+2 \varepsilon p^{\prime}(t)\right] v(z)\right\}_{i}+\frac{\partial^{2} X_{B}}{\partial t^{2}}
$$

where

$$
\begin{array}{lll}
M=k & \varepsilon=\frac{\varepsilon_{1}}{k} & \text { when } n=2 \\
M=-c & \varepsilon=\frac{\varepsilon_{0}}{k} & \text { when } n=4
\end{array}
$$

Multiplying [10] by the factor $v(z)$, using the orthogronality properties of the $v(z)$, and considering that

$$
\left.\left(\int_{0}^{l} \frac{\partial^{n} u}{\partial z^{\mathrm{n}}} \ddot{v}(z) d z\right)_{1}-l_{0}^{1} \int_{0}^{!} u \frac{\partial^{\mathrm{n}} v(z)}{\partial z^{\mathrm{n}}}\right)_{\mathrm{j}}=\left(-\lambda p \int_{\mathrm{o}}^{1} v^{2}(z) d z\right)_{\mathrm{j}}
$$

we easily obtain

$$
p^{\prime \prime}+2 \varepsilon p^{\prime}+\omega^{2} p=-\beta \frac{\partial^{2} X_{13}}{\partial t^{2}}
$$

where:

$$
\beta=\frac{\int_{0}^{1} v(z) d z}{\int_{0}^{1} v^{2}(z) d z} ; \quad \omega^{2}=\frac{\lambda}{M}
$$

When applied to $[8]$, the initial conditions require that

$$
p(t)=0 \quad p^{\prime}(t)=O \quad \text { when } \quad t=O
$$


From this and from [11] we draw the conclusion that each coeffcient $p$ represents the movement of a dynamic system initially at rest, and forced by $\beta \frac{\partial^{2} X_{\mathrm{R}}}{\partial t^{2}}$. Using Rayleigh's formulae (d) (j) we can put:

$$
p=\beta \frac{T^{\prime}}{2 \pi} \int_{0}^{t} \frac{\partial^{2} X_{\mathrm{B}}}{\partial t^{2}}(\tau) e^{-\varepsilon(t-\tau)} \operatorname{sen} \frac{2 \pi}{T^{\prime}}(t-\tau) d \tau
$$

where:

$$
T^{\prime}=\frac{2 \tau}{\sqrt{\omega^{2}-\varepsilon^{2}}}
$$

3. - Accordingr to [1], [9] and [11], it is evident that

$$
\begin{gathered}
q=\rho S\left[\sum_{\mathrm{j}}^{\infty}\left\{\left(p^{\prime \prime}+z \varepsilon \mu^{\prime}\right) v(z)\right\}_{\mathrm{y}}+\frac{\partial^{2} X_{\mathrm{B}}}{\partial \iota^{z}}\right]=-c S\left[\sum _ { j y } ^ { \infty } \left\{\left(\omega^{2} p+\right.\right.\right. \\
\left.+\beta \frac{\partial^{2} X_{\mathrm{B}}}{\partial \iota^{2}}\right)
\end{gathered}
$$

As we shall prove in detail in a further paper to be published in the "Publicaciones del Observatorio Astronómico de Eva Perón ",

$$
\sum_{j=i}^{j=i} \beta_{j} v_{j}(z)=1 \quad \text { when } O<z \leqq 1
$$

Consequently this simple expression for q remains:

$$
q=-c S \underset{j}{\stackrel{\alpha}{S}}\left(\omega^{2} p v(z)\right)_{\mathrm{j}}
$$

which shows that in order to obtain $\%$. we bave to know the function $p(t)$.

This function can be determined with the help of formulae [12]. ts $\frac{\partial \cdot X_{B}}{\partial t^{-}}$is an empirical function, the integral can he calculated only numerically or mechanically.

The first way was employed with a different aim. by A. Blake ( $\left.{ }^{6}\right)$, come years ago. Because of the work involved it is scarcely attractive. [Tse of the second way was recently made by Alford Housner and Martel $(\bar{c})$. These investigators studying the seismic action on buildings from another viewpoint determined integrals similar to 
those in [12], by means of the electrical analog computer of the California Institute of Technology, for values of $T=2 \pi / \omega$ ranging from 0.1 to 3.0 seconds and for values of $\varepsilon / \omega$ ranging from 0.0 to 0.1 . using accelerograms of several strongr earthquakes. Special attention was paid hy them to the maximum valne $X_{\mathrm{m}}$ of the integral for each $T$. With their results, they constructed curves of $X_{m}$ as a function of $T$, which they called "spectrum " of the earthquakes.

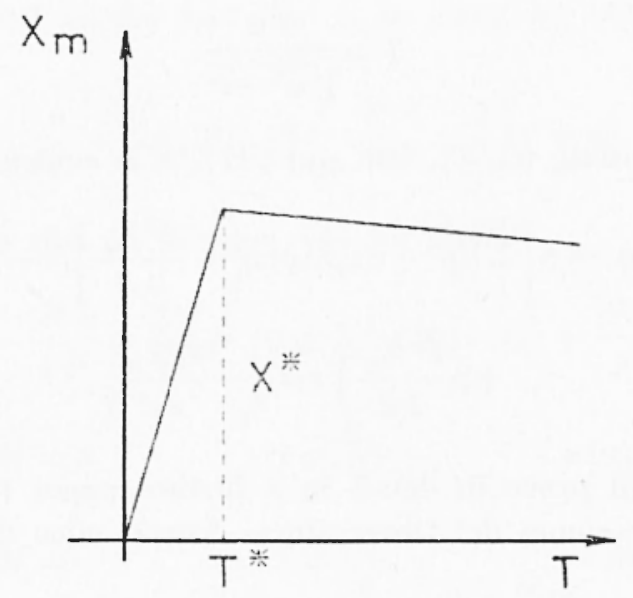

Fig. 2

Having now this spectrum at our disposal, we tried to improve the empirical estimate of seismic charges, that is customary in engineering, using values of $X_{m}$ instead of the accurate values of the integrals written in [12]. With this, and the assumptions that $T^{\prime}=2 \pi / \omega$ we olstain instead of [14]

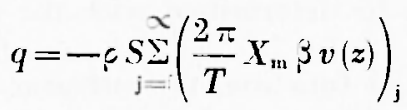

Curves representing $X_{\mathrm{m}}$ are also complicated. But it is possible to aproximate them with simpler ones and try with these to ohtain the sum pointed out in [14].

It is very interesting to note that, if we adopt for $X_{m}$ the representation given in fig. 2 (which can be obtained by smoothing the 
true curves! then for huildings in which the maximum $T$ is less than $T^{*}$, we get from [15] that:

$$
q=-\rho S \frac{2 \pi X^{*}}{I^{*}} \Sigma \beta v(z)
$$

or remembering $[13]$ :

$$
q=-p s \frac{2 \pi X^{*}}{T^{*}}
$$

We arrive thus to the remarkable conclusion that the seismic rharge $q$ should be constant all along the building; a fact that is customarily accepted among engineers, whithout demonstration.

From measurements made in the U.S.A. $\left({ }^{8}\right)$ we have deduced that

$$
T=0,02 \quad l
$$

$l=$ height of the buildings in metres

$$
T=\text { in seconds }
$$

According to the refered spectrum, $T^{*}$ is of the order of 0,6 seconds. Consequently the assumption that $q$ is constant could be accurate for buildings less than $30 \mathrm{~m}$ height. For higher huildings it can be proved that $q$ decreases with $l$.

Observatorio Astronomico

Eva Perón (formerly La Plata), May 1954

\section{$S U M M A R Y$}

Deformations " of buildings due to the acceleration of earthquakes are investigated. Tuo suppositions are made: al the deformations are principally due to shear; and b) they are principally due to bending.

In both cases it is found that $u$ may be expressed by an uniformly convergent series of eigenfunctions.

Except for a variable factor, the Fourier coefficients of these series are the same as the values $X$ obtained recently by Alford Housner and Martel, by means of the electrical analog computer of the California Institute of Technology. 
Lising mean values of what they call the spectrum of earthquakes, it is found that the seismic charge is constant on buildings less than $30 \mathrm{~m}$ height. On buildings higher than $30 \mathrm{~m}$ it becomes decreasing with height.

\section{RIASSUNTO}

Iengono studiate le deformazioni di edifici, dovate allaccelerazione determinata da terremoti. Vengono fatte due ipotesi: a) le deformazioni sono principalmente dovute a forze tangenziali; b) esse sono principalmente legate alla flessione.

In entrambi i casi, si prova che esse possono venir espresse mediante una serie uniformemente convergente di autofunzioni.

Fatta eccezione di un fattore variabile, $i$ coefficienti di lourier di questa serie sono gli stessi che figurano nei valori ottenuti da .llford Housner e Martel in ricerche su argomenti analoghi condotte presso il "California Institute of Technology".

Facendo uso dei valori medi del cosi detto spettro dei terremoti, l'Autore trova che la carica sismica su edifici di altezza minore di 30 metri è costante, mentre tende a diminuire per altezze maggiori.

\section{REFERENCES}

(1) Cocnaxt Hibbent, Methoden der Mathematischen Physik, I Kap V 10, 14.

(2) L. CoLLATz. Eigenwertauggaben mit Technischen anwendungen, Kap II, 5.

(3) Counant Hilbert, loc. cit., Kap. III $5,1$.

(4) Idem, Kap Y 2, 3.

(5) Lord Rayleigh, Theory of Sound, Chap II 66, Dover Publ. 19.15.

(6) A. Blake, Proceedings of the 1935, Meeting Eastern Sec Seism. Soc. America.

(i) Alfond Housner and Martel. Bull. Seismological Soc. of America, vol. 13, Number 2.

(8) U. S. Cosst and Geobetic Stnvey, Earthquake investigations in California 1934-1935, Special publication, n. 201. 